\title{
Target scattering estimation in clutter with polarization optimization
}

\author{
Xu Cheng*, Longfei Shi, Yuliang Chang, Yongzhen Li and Xuesong Wang
}

\begin{abstract}
In this paper, we propose an adaptive waveform polarization method for the estimation of target scattering matrix in the presence of clutter. The proposed sequential algorithm, based on the concept of sequential minimum mean square error (MSE) estimation, to determine the coefficients of the scattering matrix, guarantees the convergence and the resulting computational complexity is linear with the number of iterations. The effectiveness of the proposed method is validated through numerical results, underlining the performance improvement given by joint transmission and reception (Tx/Rx) polarization optimization for the scalar system. Also, the results show that the vector system with transmission polarization optimization provides a comparative performance as the scalar measurement system employing joint Tx/Rx polarization optimization. Less computation burden highlights the advantage of the former mode.
\end{abstract}

Keywords: Radar polarization, Minimum MSE estimation, Sequential algorithm, Waveform design, Scattering matrix

\section{Introduction}

Polarization, together with the amplitude, time, frequency, phase, and bearing descriptor of radar signals, completes the information description of target returns from radars. The exploitation of information on the echo polarization can provide a significant improvement on radar performance [1]. To obtain the target polarimetric scattering information, conventional polarimetric radar systems alternately switch (or simultaneously transmit) the horizontal $(\mathrm{H})$ and vertical $(\mathrm{V})$ polarizations at the transmission side, and simultaneously receive both polarizations at the reception side, consequently resulting in four polarization combinations: $\mathrm{HH}, \mathrm{HV}, \mathrm{VH}$, and $\mathrm{VV}$ polarizations.

Motivated in part by recent advances in the hardware and sensor information processing, such as solid state transmission and digital arbitrary waveform generators, in modern radar systems, any polarization on either transmission or reception can be synthesized by using the linear combination of the $\mathrm{H}$ and $\mathrm{V}$ components.

\footnotetext{
* Correspondence: chengxu@nudt.edu.cn

State Key Laboratory of Complex Electromagnetic Environment Effects on Electronics and Information System, College of Electronic Science and Engineering, National University of Defense Technology, Changsha 410073, People's Republic of China
}

Thus, besides the four types of transmitter/receiver combinations above, polarimetric radar can achieve any pair of transmitter/receiver polarizations. Such flexibility greatly enhances the polarimetric sensing capability of the radar system. For the mentioned reason, several papers concerning polarization optimization have appeared in the open literature during the last two decades aiming at the performance of polarimetric radars on target estimation [2,3], detection [4-8], tracking [9], and identification $[4,10]$.

This paper will tackle with the problem of adaptively selecting waveform polarization to optimally estimate the target scattering matrix. As to this topic, the technique of optimizing transmission polarization for "vector measurement systems" [10] (with the term "vector measurement system", the authors refer to the reception side of the radar that receives separately the horizontal and vertical polarization components to form a vector) has been addressed in [2]. The numerical results there show that, by optimally selecting waveform polarization, the performance of the target estimation can be significantly improved compared to the fixed polarization design. In most cases, polarimetric radar systems combine two received signals linearly and coherently at the receiver to give a scalar measurement [1]. Following [6], we call the radar

\section{Springer}


employing such measurement mode as the "scalar measurement system". As to this kind of system, in [3], the authors devise an optimization algorithm to jointly design $\mathrm{Tx} / \mathrm{Rx}$ polarizations that minimize the MSE of estimating the target scattering vector. The original problem therein is formulated into a convex form which is solvable employing semi-definite programming (SDP). As a subfield of convex optimization, SDP concerns with the optimization of a linear objective function to be maximized or minimized over the intersection of the cone of positive semi-definite matrices with an affine space, i.e., a spectrahedron [11]. Such optimization problems can be solved via the well-known CVX toolbox for Matlab software [12]. But unfortunately, we find the problem formulation in [3] cannot be effectively solved by exploiting the devised algorithm (see Appendix 1 for details). In fact, the SDP formulation there is a relaxation version of the original problem to be handled.

Hence, in this paper, we reconsider the problem of adaptive polarization design of polarimetric radars to optimally estimate the target scattering vector in clutter. Starting from scalar measurement systems, we design an optimization procedure for the $\mathrm{Tx} / \mathrm{Rx}$ polarizations to sequentially minimize the MSE of estimating the target scattering vector. The proposed algorithm converges to a certain value, and the computational complexity is linear with the number of iterations. Furthermore, we generalize the algorithm to the vector measurement system to optimally design the transmission polarization. At the analysis stage, we validate the effectiveness of the proposed method through numerical results, highlighting the performance improvement of the joint $\mathrm{Tx} / \mathrm{Rx}$ polarization optimization for the scalar system. Our results show that the vector system with transmission polarization optimization offers a comparative performance as the scalar system employing joint $\mathrm{Tx} / \mathrm{Rx}$ polarization optimization.

The remainder of the paper is organized as follows. The measurement model of the scalar measurement system with jointly adaptive $\mathrm{Tx} / \mathrm{Rx}$ polarization design is introduced in Section 2. In Section 3, the problem formulation is described. The sequential method to optimally select waveform parameter with affordable computation burden is proposed in Section 4. Then, in Section 5, the devised algorithm is exploited to the vector measurement system. Numerical results are presented in Section 6, demonstrating the improvement in performance achieved by using the joint $\mathrm{Tx} /$ $\mathrm{Rx}$ polarization in comparison with the two other scalar measurement systems, as well as highlighting the advantage of vector measurement systems with respect to scalar ones. Finally, the conclusions are provided in Section 7.

\section{Measurement model}

In this section, we formulate the parametric measurement model of target scattering vector in additive Gaussian distributed clutter for the scalar measurement system with adaptive $\mathrm{Tx} / \mathrm{Rx}$ polarization designs.

Let $\boldsymbol{\xi}=\left[\xi_{\mathrm{h}}, \xi_{\mathrm{v}}\right]^{\mathrm{T}}$ be the transmitted polarized electric field impinging on the target and $\boldsymbol{\eta}=\left[\eta_{\mathrm{h}}, \eta_{\mathrm{v}}\right]^{\mathrm{T}}$ be the reception antenna polarization, where the subscripts "h" and "v" denote the horizontal and vertical polarization components of a fully polarized signal, respectively, and the superscript " $T$ " denotes the transpose operation. We consider that the transmission pulse has unitary power, i.e., $\|\mathcal{E}\|=1$. As to the reception polarization, we set $\|\eta\|=1$. After ignoring the target Doppler shift, the complex envelope of the received signal is given by $[3,5]$

$$
y(t)=\frac{g}{r^{2}} \boldsymbol{\eta}^{\mathrm{T}}\left(\boldsymbol{S}_{\mathrm{t}}+\boldsymbol{S}_{\mathrm{c}}\right) \boldsymbol{\xi} s(t-\tau)+w(t)
$$

where $w(t)$ is the white noise, $r$ is the distance from the target to radar, $s(t)$ is the transmission waveform, $\tau$ is the delay resulted from the waveform forward and backward propagation, and $g$ is a constant depending on the radar system characteristics including operating frequency, permittivity, permeability of free space, antenna gain at the target illumination angle, radar reception power, etc. $S_{\mathrm{t}}$ and $S_{\mathrm{c}}$ are the target and clutter scattering matrices, respectively, with the following matrix representation:

$$
S_{\mathrm{t}}=\left[\begin{array}{cc}
s_{\mathrm{hh}}^{\mathrm{t}} & s_{\mathrm{hv}}^{\mathrm{t}} \\
s_{\mathrm{vh}}^{\mathrm{t}} & s_{\mathrm{vv}}^{\mathrm{t}}
\end{array}\right], \quad \boldsymbol{S}_{\mathrm{c}}=\left[\begin{array}{cc}
s_{\mathrm{hh}}^{\mathrm{c}} & s_{\mathrm{hv}}^{\mathrm{c}} \\
s_{\mathrm{vh}}^{\mathrm{c}} & s_{\mathrm{vv}}^{\mathrm{c}}
\end{array}\right] .
$$

Here, we remark that the linear assumption has been employed to linearize the electromagnetic scattering problem. From a more general point of view, the electromagnetic scattering problem is actually highly nonlinear. But the linearity assumption is usually employed to simplify the problem at hand. In this paper, we make the linearity assumption following $[3,5,9]$, to address the similar signal model.

After performing the sampling process and matched filtering [13], (1) could be expressed in the following observation model:

$$
y=\boldsymbol{\eta}^{\mathrm{T}}\left(\boldsymbol{S}_{\mathrm{t}}+\boldsymbol{S}_{\mathrm{c}}\right) \boldsymbol{\xi}+v,
$$

where $v$ is the noise with variance $\sigma_{v}^{2}$. By vectorizing $S_{\mathrm{t}}$ and $S_{\mathrm{c}}$, we could convert (3) into the observation model 


$$
y=\boldsymbol{a}(\boldsymbol{\xi}, \boldsymbol{\eta})^{T} \boldsymbol{X}_{\mathrm{t}}+\boldsymbol{a}(\boldsymbol{\xi}, \boldsymbol{\eta})^{T} \boldsymbol{X}_{\mathrm{c}}+v,
$$

where

$$
\begin{aligned}
& \boldsymbol{X}_{\mathrm{t}}=\left[s_{\mathrm{hh}}^{\mathrm{t}} s_{\mathrm{hv}}^{\mathrm{t}} s_{\mathrm{vh}}^{\mathrm{t}} s_{\mathrm{vv}}^{\mathrm{t}}\right]^{\mathrm{T}},
\end{aligned}
$$

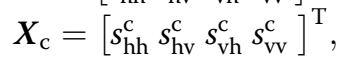

and

$$
\boldsymbol{a}(\boldsymbol{\xi}, \boldsymbol{\eta})=\left[\xi_{\mathrm{h}} \eta_{\mathrm{h}} \xi_{\mathrm{h}} \eta_{\mathrm{v}} \xi_{\mathrm{v}} \eta_{\mathrm{h}} \xi_{\mathrm{v}} \eta_{\mathrm{v}}\right]^{\mathrm{T}} .
$$

Assuming that there are $N$ pulses with different polarizations used to estimate the fully polarimetric target information $\boldsymbol{X}_{\mathrm{t}}$, the observation from these $N$ pulses can be written as

$$
y(i)=\boldsymbol{a}(\boldsymbol{\xi}(i), \boldsymbol{\eta}(i))^{\mathrm{T}} \boldsymbol{X}_{\mathrm{t}}+\boldsymbol{a}(\boldsymbol{\xi}(i), \boldsymbol{\eta}(i))^{\mathrm{T}} \boldsymbol{X}_{\mathrm{c}}+v(i), \quad i=1, \ldots, N,
$$

where $\boldsymbol{\xi}(i)$ and $\boldsymbol{\eta}(i)$ denote, respectively, the transmission and reception polarizations of the $i$ th pulse, i.e., $\boldsymbol{a}$ $(\boldsymbol{\xi}(i), \boldsymbol{\eta}(i))=\left[\xi_{\mathrm{h}}^{(i)} \eta_{\mathrm{h}}^{(i)} \xi_{\mathrm{h}}^{(i)} \eta_{\mathrm{v}}^{(i)} \xi_{\mathrm{v}}^{(i)} \eta_{\mathrm{h}}^{(i)} \xi_{\mathrm{v}}^{(i)} \eta_{\mathrm{v}}^{(i)}\right]^{\mathrm{T}}$. For notational simplicity, we define $\boldsymbol{a}(i) \triangleq \boldsymbol{a}(\boldsymbol{\xi}(i), \boldsymbol{\eta}(i))$. Introducing further the following vector notations

$$
\begin{aligned}
& \mathbf{y}=[y(1), \ldots, y(N)]^{\mathrm{T}}, \\
& \mathbf{A}=[\boldsymbol{a}(1), \ldots, \boldsymbol{a}(N)]^{\mathrm{T}}, \\
& \mathbf{v}=[v(1), \ldots, v(N)]^{\mathrm{T}},
\end{aligned}
$$

we obtain the matrix representation of the linear measurement model as

$$
\mathbf{y}=\mathbf{A} X_{\mathrm{t}}+\mathbf{A} \boldsymbol{X}_{\mathrm{c}}+\mathbf{v} .
$$

Notice that the system response $\mathbf{A}$ here is an $N \times 4$ complex matrix; so we assume that $N>4$ and ensure $\operatorname{rank}(\mathbf{A})=4$ to estimate four-dimensional complex vector $\boldsymbol{X}_{\mathrm{t}}[3,5]$.

As to the target and clutter statistical characterizations, we do not make restrictive model assumption on the multivariate statistical characterization of $X_{\mathrm{t}}$ and $\boldsymbol{X}_{\mathrm{c}}$. However, we assume that we have access to the first two moments of their probability density function (PDF). This assumption is reasonable, especially in a knowledge-aided (possibly cognitive) radar scenario. In such case, clutter statistical parameters can be obtained jointly using geographical information, meteorological measurements, and statistical (possibly empirical) models for the clutter [14]. The statistical parameters of the illuminated target can be obtained or roughly estimated by pre-scan procedures and employing cognitive methods [14-16]. Precisely, we assume $\boldsymbol{X}_{\mathrm{t}}$ is a four-dimensional random vector of parameters whose realization is to be estimated and has mean $E\left(\boldsymbol{X}_{\mathrm{t}}\right)$ and covariance matrix $\boldsymbol{C}_{\mathrm{t}}$, and $\boldsymbol{X}_{\mathrm{c}}$ is a four-dimensional random vector with zero mean and covariance matrix $C_{\mathrm{c}}$ and is uncorrelated with $\boldsymbol{X}_{\mathrm{t}}$, where $E(\cdot)$ denotes the expectation operation. As to the statistical characterization of the noise vector, we assume that it is Gaussian white with covariance matrix $\sigma_{v}^{2} \mathbf{I}_{4}$, where $\mathbf{I}_{4}$ is the identity matrix with a $4 \times 4$ size. Finally, we assume that the target, clutter, and noise are uncorrelated, so that the joint PDF $p$ $\left(\boldsymbol{X}_{\mathrm{t}}, \boldsymbol{X}_{\mathrm{c}}, \mathbf{v}\right)$ is arbitrary.

\section{Problem formulation}

Our assignment is to estimate the target scattering vector $\boldsymbol{X}_{\mathrm{t}}$ from the observations. By inspection of the measurement model in the previous section, it matches the Bayesian linear model form. By exploiting the Bayesian Gauss-Markov Theorem [[17], Theorem 12.1], we obtain the minimum MSE estimator of $\boldsymbol{X}_{\mathrm{t}}$ as

$$
\hat{\boldsymbol{X}}_{\mathrm{t}}=E\left(\boldsymbol{X}_{\mathrm{t}}\right)+\boldsymbol{C}_{\mathrm{t}} \mathbf{A}^{\mathrm{H}}\left(\mathbf{A}\left(\boldsymbol{C}_{\mathrm{t}}+\boldsymbol{C}_{\mathrm{c}}\right) \mathbf{A}^{\mathrm{H}}+\sigma_{v}^{2} \mathbf{I}_{N}\right)^{-1}\left(\mathbf{y}-\mathbf{A} E\left(\boldsymbol{X}_{\mathrm{t}}\right)\right),
$$

where $\mathbf{I}_{N}$ is the identity matrix with a $N \times N$ size and $(\cdot)^{\mathrm{H}}$ denotes the conjugate transpose. The performance of the estimator is measured by the error $\boldsymbol{e}=\boldsymbol{X}_{\mathrm{t}}-\hat{\boldsymbol{X}}_{\mathrm{t}}$ whose mean value is zero and covariance matrix is

$$
\boldsymbol{D}=\left(\boldsymbol{C}_{\mathrm{t}}^{-1}+\mathbf{A}^{\mathrm{H}}\left(\mathbf{A} \boldsymbol{C}_{\mathrm{c}} \mathbf{A}^{\mathrm{H}}+\sigma_{v}^{2} \mathbf{I}_{N}\right)^{-1} \mathbf{A}\right)^{-1},
$$

with the $i$ th diagonal element of $\boldsymbol{D}$ as the minimum Bayesian MSE of $\boldsymbol{X}_{\mathrm{t}}^{\prime}$ 's $i$ th element [Kay [17], Theorem 12.1]. Hence, the trace of $D$, i.e., $\operatorname{Tr}(\boldsymbol{D})$, represents the sum of the minimum Bayesian MSEs of all $X_{\mathrm{t}}$ 's four elements. As a consequence, we define the MSE of $X_{\mathrm{t}}$ as $\operatorname{Tr}(\boldsymbol{D})$. As done in [3], we consider $\operatorname{Tr}(\boldsymbol{D})$ as the relevant figure of merit and minimize it to optimize transmission and reception polarizations.

Thus, the problem of jointly optimizing transmission and reception polarizations, to minimize the MSE of estimating $\boldsymbol{X}_{\mathrm{t}}$ with $N$ diversely polarized pulses, can be formulated as

$$
\mathcal{P}\left\{\begin{array}{c}
\min _{\boldsymbol{D},\{\boldsymbol{\xi}(i)\}_{i=1}^{N},\{\boldsymbol{\eta}(i)\}_{i=1}^{N}} \operatorname{Tr}(\boldsymbol{D}) \\
\text { s.t. } \boldsymbol{D}=\left(\boldsymbol{C}_{\mathrm{t}}^{-1}+\mathbf{A}^{\mathrm{H}}\left(\mathbf{A} \boldsymbol{C}_{\mathrm{c}} \mathbf{A}^{\mathrm{H}}+\sigma_{v}^{2} \mathbf{I}_{N}\right)^{-1} \mathbf{A}\right)^{-1} \text { with } \\
\mathbf{A}=[\boldsymbol{a}(1), \ldots, \boldsymbol{a}(N)]^{\mathrm{T}} \text { with } \\
\boldsymbol{a}(i)=\left[\xi_{\mathrm{h}}^{(i)} \eta_{\mathrm{h}}^{(i)}, \xi_{\mathrm{h}}^{(i)} \eta_{\mathrm{v}}^{(i)}, \xi_{\mathrm{v}}^{(i)} \eta_{\mathrm{h}}^{(i)}, \mathcal{\xi}_{\mathrm{v}}^{(i)} \eta_{\mathrm{v}}^{(i)}\right]^{\mathrm{T}} \text { with } \\
\boldsymbol{\xi}(i)=\left[\xi_{\mathrm{h}}^{(i)}, \mathcal{\xi}_{\mathrm{v}}^{(i)}\right]^{\mathrm{T}} \text { with }\|\boldsymbol{\xi}(i)\|=1, \\
\boldsymbol{\eta}(i)=\left[\eta_{\mathrm{h}}^{(i)}, \eta_{\mathrm{v}}^{(i)}\right]^{\mathrm{T}} \text { with }\|\boldsymbol{\eta}(i)\|=1, i=1, \ldots, N .
\end{array}\right.
$$

Remarks Actually, there exist several potential optimality criteria for the problem at hand, e.g., minimizing the determinant of $D$, minimizing the maximum eigenvalue of $\boldsymbol{D}$ and the aforementioned $\operatorname{Tr}(\boldsymbol{D})$, etc. [19]. In this paper, we employ $\operatorname{Tr}(\boldsymbol{D})$ because of the 
following: firstly, it is reasonable, as stated before and secondly, we exploit the same problem formulation as in [3] and then highlight our design.

\section{Optimal waveform selection}

In the sequel, we will deal with the non-convex (the objective is non-convex and $\|\boldsymbol{\xi}(i)\|=\|\boldsymbol{\eta}(i)\|=1$ defines a non-convex set) optimization problem $\mathcal{P}$. By reformulating the problem using trigonometric parameters and the inspection of its computational complexity, we exploit the sequential minimum MSE estimation scheme to compute the optimal $\mathrm{Tx} / \mathrm{Rx}$ polarizations instead of directly utilizing the minimum MSE estimation.

\subsection{Problem reformulation}

A first step toward the goal to tackle the optimization problem is represented by the following notations of transmission and reception polarizations. As to the transmission polarization, it can be parameterized into the following trigonometric function [2]:

$$
\boldsymbol{\xi}=\|\boldsymbol{\xi}\| \mathrm{e}^{j \varphi} Q \boldsymbol{w}
$$

where $Q=\left[\begin{array}{cc}\cos \alpha & \sin \alpha \\ -\sin \alpha & \cos \alpha\end{array}\right], \boldsymbol{w}=\left[\begin{array}{c}\cos \beta \\ j \sin \beta\end{array}\right],\|\mathcal{E}\| e^{j \phi}$ is the complex envelope of the source signal, $\alpha$ denotes the rotation angle between the system coordinates and the electric ellipse axes, and $\beta$ determines the ellipse's eccentricity, respectively, with the definition spaces of these trigonometric parameters being $\phi \in(-\pi, \pi], \alpha \in[-\pi / 2, \pi / 2]$, and $\beta \in[-\pi / 4, \pi / 4]$. Since the transmitted signal is power limited, i.e., $\|\xi\|=1$, by replacing $Q$ and $\boldsymbol{w}$ with their explicit forms, we can rewrite (13) as

$$
\boldsymbol{\xi} \triangleq \mathrm{e}^{j \varphi}\left[\begin{array}{c}
\zeta_{\mathrm{h}} \\
\zeta_{\mathrm{v}}
\end{array}\right]=\mathrm{e}^{j \varphi}\left[\begin{array}{c}
\cos \alpha \cos \beta+j \sin \alpha \sin \beta \\
-\sin \alpha \cos \beta+j \cos \alpha \sin \beta
\end{array}\right]
$$

Similarly, the trigonometric form of received polarization can be written as

$$
\boldsymbol{\eta} \triangleq \mathrm{e}^{j \phi}\left[\begin{array}{c}
\iota_{\mathrm{h}} \\
\iota_{\mathrm{v}}
\end{array}\right]=\mathrm{e}^{j \phi}\left[\begin{array}{c}
\cos \theta \cos \vartheta+j \sin \theta \sin \vartheta \\
-\sin \theta \cos \vartheta+j \cos \theta \sin \vartheta
\end{array}\right]
$$

where $\phi \in(-\pi, \pi], \theta \in[-\pi / 2, \pi / 2]$, and $\vartheta \in[-\pi / 4, \pi / 4]$.

We can observe that, once $\boldsymbol{\xi}$ and $\boldsymbol{\eta}$ are written into (14) and (15), respectively, $\operatorname{Tr}(\boldsymbol{D})$ can be uniquely determined by trigonometric parameters $\{\phi, \alpha, \beta, \phi, \theta, \vartheta\}$. Before proceeding further, herein we introduce an interesting observation of $\operatorname{Tr}(\boldsymbol{D})$ with respect to such parameters, as summarized in following property.

Property 1 The initial signal phases $\phi$ and $\phi$ do not affect the value of $\boldsymbol{D}$.

Proof See Appendix 2.

It can be seen that with Property $1, \operatorname{Tr}(\boldsymbol{D})$ can be uniquely determined by four trigonometric parameters $\{\alpha, \beta, \theta, \vartheta\}$ instead of six trigonometric parameters $\{\phi, \alpha$, $\beta, \phi, \theta, 9\}$. Thus, the problem $\mathcal{P}$ can be equivalently recast as

$$
\mathcal{P}_{1}\left\{\begin{array}{c}
\min _{\boldsymbol{D},\left\{\alpha_{i}\right\}_{i=1}^{N},\left\{\beta_{i}\right\}_{i=1}^{N},\left\{\theta_{i}\right\}_{i=1}^{N},\left\{\vartheta_{i}\right\}_{i=1}^{N}} \operatorname{Tr}(\boldsymbol{D}) \\
\text { s.t. } \boldsymbol{D}=\left(\boldsymbol{C}_{\mathrm{t}}^{-1}+\mathbf{A}^{\mathrm{H}}\left(\mathbf{A} \boldsymbol{C}_{\mathrm{c}} \mathbf{A}^{\mathrm{H}}+\sigma_{v}^{2} \mathbf{I}_{N}\right)^{-1} \mathbf{A}\right)^{-1} \text { with } \\
\mathbf{A}=[\boldsymbol{a}(1), \ldots, \boldsymbol{a}(N)]^{\mathrm{T}} \text { with } \\
\boldsymbol{a}(i)=\left[\xi_{\mathrm{h}}^{(i)} \eta_{\mathrm{h}}^{(i)}, \xi_{\mathrm{h}}^{(i)} \eta_{\mathrm{v}}^{(i)}, \xi_{\mathrm{v}}^{(i)} \eta_{\mathrm{h}}^{(i)}, \xi_{\mathrm{v}}^{(i)} \eta_{\mathrm{v}}^{(i)}\right]^{\mathrm{T}} \text { with } \\
{\left[\begin{array}{c}
\xi_{\mathrm{h}}^{(i)} \\
\xi_{\mathrm{v}}^{(i)}
\end{array}\right]=\left[\begin{array}{c}
\cos \alpha_{i} \cos \beta_{i}+j \sin \alpha_{i} \sin \beta_{i} \\
-\sin \alpha_{i} \cos \beta_{i}+j \cos \alpha_{i} \sin \beta_{i}
\end{array}\right] \text { and }} \\
{\left[\begin{array}{l}
\eta_{\mathrm{h}}^{(i)} \\
\eta_{\mathrm{v}}^{(i)}
\end{array}\right]=\left[\begin{array}{c}
\cos \theta_{i} \cos \vartheta_{i}+j \sin \theta_{i} \sin \vartheta_{i} \\
-\sin \theta_{i} \cos \vartheta_{i}+j \cos \theta_{i} \sin \vartheta_{i}
\end{array}\right] \text { with }} \\
\alpha_{i} \in[-\pi / 2, \pi / 2], \beta_{i} \in[-\pi / 4, \pi / 4] \\
\theta_{i} \in[-\pi / 2, \pi / 2], \vartheta_{i} \in[-\pi / 4, \pi / 4], i=1, \ldots, N .
\end{array}\right.
$$

We can see that $\mathcal{P}_{1}$ is still a non-convex optimization problem because the objective function is the same non-convex function as in $\mathcal{P}$. Nevertheless, as opposed to $\mathcal{P}$, the equivalent formulation provided by $\mathcal{P}_{1}$ shows that lattice search along the trigonometric parameters $\{\alpha, \beta, \theta, \vartheta\}$ can be employed to calculate the optimal polarization, as done in [2] and [5]. But since lattice search does not explore any optimization property, it requires a high computational burden. Precisely, the computational complexity is firstly exponential to the observation number $N$. Also, it highly depends on the maximum point search algorithm used for $\{\alpha, \beta, \theta, \vartheta\}$. If we use lattice search with $l_{\alpha}, l_{\beta}, l_{\theta}$ and $l_{\vartheta}$ points in each dimension of the domain space $\{\alpha, \beta, \theta, 9\}$, respectively, the relevant complexity burden is $O\left(\left(l_{\alpha} l_{\beta} l_{\theta} l_{9}\right)^{N}\right)$.

\subsection{Sequential minimum MSE estimation}

Since to solve $\mathcal{P}_{1}$ straightforward requires a high computational burden, we exploit the sequential minimum MSE estimation to tackle it. By insight of $\mathcal{P}_{1}$, we can observe that it works at the case of block signal polarization design, namely designing the polarizations of $N$ consecutive temporal signal samples at a time. However, in real radar signal processing applications, the returns are ongoing as time progresses. So it is reasonable to process the data sequentially in time. As to the problem at hand, the resulting solution belongs to the sequential minimum MSE estimation. Thus, by following the sequential minimum MSE estimation procedure in [Kay [17], Eq. (12.47)-(12.49)], we develop the sequential minimum MSE estimation to handle $\mathcal{P}_{1}$ as follows.

Let $\hat{\boldsymbol{X}}_{\mathrm{t}, n}$ denote the minimum MSE estimator based on the observations $[y(1), \ldots, y(n)]^{\mathrm{T}}$ and $\boldsymbol{D}_{n}$ be the 
corresponding minimum MSE matrix (just the sequential version of (10) and (11)). Then, when the new sample $y(n+1)$ is available, the estimator is updated as

$$
\hat{\boldsymbol{X}}_{\mathrm{t}, n+1}=\hat{\boldsymbol{X}}_{\mathrm{t}, n}+\boldsymbol{K}_{n+1}\left(y(n+1)-\boldsymbol{a}^{\mathrm{T}}(n+1) \hat{\boldsymbol{X}}_{\mathrm{t}, n}\right),
$$

where

$$
\boldsymbol{K}_{n+1}=\frac{\boldsymbol{D}_{n} \boldsymbol{a}(n+1)}{\left[\mathbf{A}_{n+1} \boldsymbol{C}_{\mathrm{c}} \mathbf{A}_{n+1}^{\mathrm{H}}+\sigma_{v}^{2} \mathbf{I}_{n+1}\right]_{n+1, n+1}+\boldsymbol{a}^{\mathrm{H}}(n+1) \boldsymbol{D}_{n} \boldsymbol{a}(n+1)},
$$

is the gain factor weighting confidence in the new data with $\mathbf{A}_{n+1}=[\boldsymbol{a}(1), \ldots, \boldsymbol{a}(n+1)]^{\mathrm{T}}$ and $\boldsymbol{a}(i)$ having the same definition as before and $[\cdot]_{n+1, n+1}$ denoting the $n+1$ th diagonal element of matrix [.]. In the meantime, the minimum MSE matrix is updated as

$$
\boldsymbol{D}_{n+1}=\left(\mathbf{I}_{n+1}-\boldsymbol{K}_{n+1} \boldsymbol{a}^{\mathrm{T}}(n+1)\right) \boldsymbol{D}_{n} .
$$

As to $D_{n+1}$, based on [Kay [17], p. 393], with $n$ increasing, $\left[\boldsymbol{D}_{n+1}\right]_{i i}, i=1,2,3,4$, decreases and converges to a certain value. Hence, $\operatorname{tr}\left(\boldsymbol{D}_{n+1}\right)$ is also a monotonic decreasing sequence and converges to a certain value.

\subsection{Optimal waveform parameter selection}

By employing the sequential minimum MSE estimation procedure described earlier, our goal is to search for the optimal $\mathrm{Tx} / \mathrm{Rx}$ polarizations for every iteration, under the criterion of minimizing the Bayesian MSE-based cost function $\operatorname{tr}\left(\boldsymbol{D}_{n}\right)$. As to each iteration, we still employ lattice search to compute the optimal $\mathrm{Tx} / \mathrm{Rx}$ polarizations. Precisely, the sequential procedure to update the optimal polarizations is summarized as Algorithm 1. Also, Fig. 1 exhibits a pictorial representation of the sequential estimation processing.
Now let us examine the computational complexity of Algorithm 1. Let us still consider that there are $N$ observation samples. Different from solving $\mathcal{P}_{1}$, Algorithm 1 is linear to the observation number $N$, with requiring the lattice search for the optimal polarizations in the domain space $\{\alpha, \beta, \theta, \vartheta\}$ in each iteration. Thus, the overall complexity is $O\left(l_{\alpha} l_{\beta} l_{\theta} l_{g} N\right)$. In comparison with the direct lattice search to solve $\mathcal{P}_{1}$, the computation burden is greatly reduced. It is not surprising since the proposed algorithm is sequential, namely it does not optimize for all observations and thus, it is clearly less expensive than the one proposed in (16).

\section{Vector measurement system}

Vector sensors, which employ two-dimensional sensors to measure both horizontal and vertical components of the electric field at each of the receivers, provide a significant improvement in performance over scalar sensors for a variety of applications $[10,18]$. For such mentioned reason, in this section, we develop the approach to optimize transmission polarization of vector measurement systems by following the similar way of designing the optimal $\mathrm{Tx} / \mathrm{Rx}$ polarizations for the aforementioned scalar system. In general, the derivation of such system model and the corresponding adaptive design process follow the similar way as the model in Section 2, so it is simplified.

\subsection{Vector measurement model}

As to the vector measurement system with adaptive transmission polarization, the transmission vector $\left[\xi_{\mathrm{h}}, \xi_{\mathrm{v}}\right]^{\mathrm{T}}$ is allowed to be chosen freely while the reception side has both horizontal and vertical channels to separately receive horizontal and vertical polarizations of the return, respectively, to form a two-dimensionalvector. The scattered field is related to the incident by

$$
\boldsymbol{y}(t)=\frac{g}{r^{2}}\left(\boldsymbol{S}_{\mathrm{t}}+\boldsymbol{S}_{\mathrm{c}}\right) \boldsymbol{\xi} s(t-\tau)+\boldsymbol{w}(t),
$$

where $\boldsymbol{y}(t)=\left[\begin{array}{ll}y_{\mathrm{h}}(t) & y_{\mathrm{v}}(t)\end{array}\right]^{\mathrm{T}}$ is the complex envelope vector of the received electric field, $\boldsymbol{w}(t)=\left[w_{\mathrm{h}}(t) w_{\mathrm{v}}(t)\right]^{\mathrm{T}}$ is the

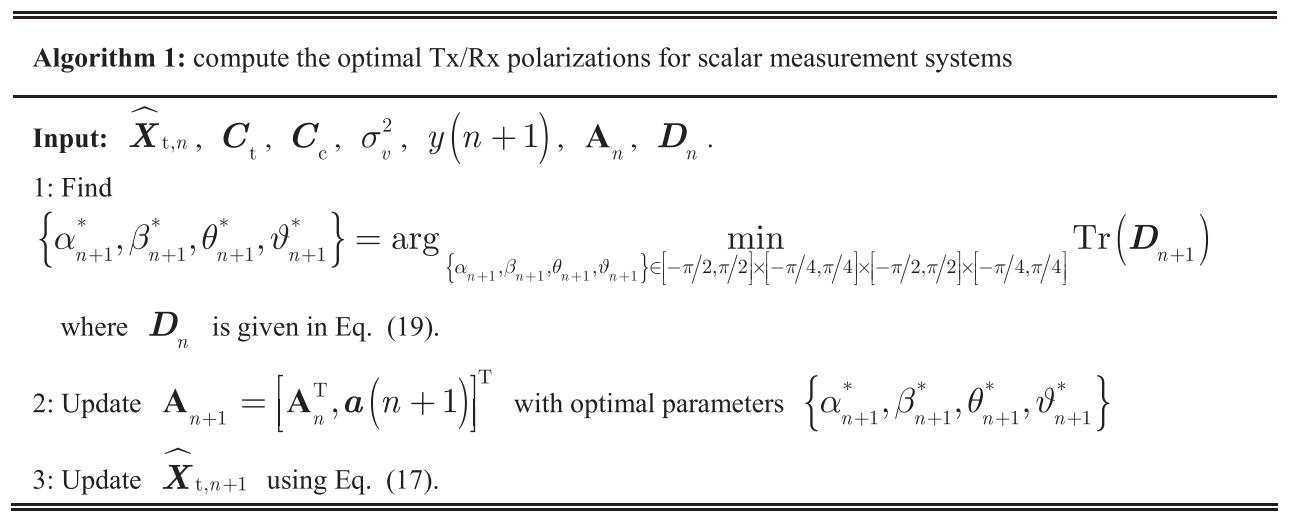




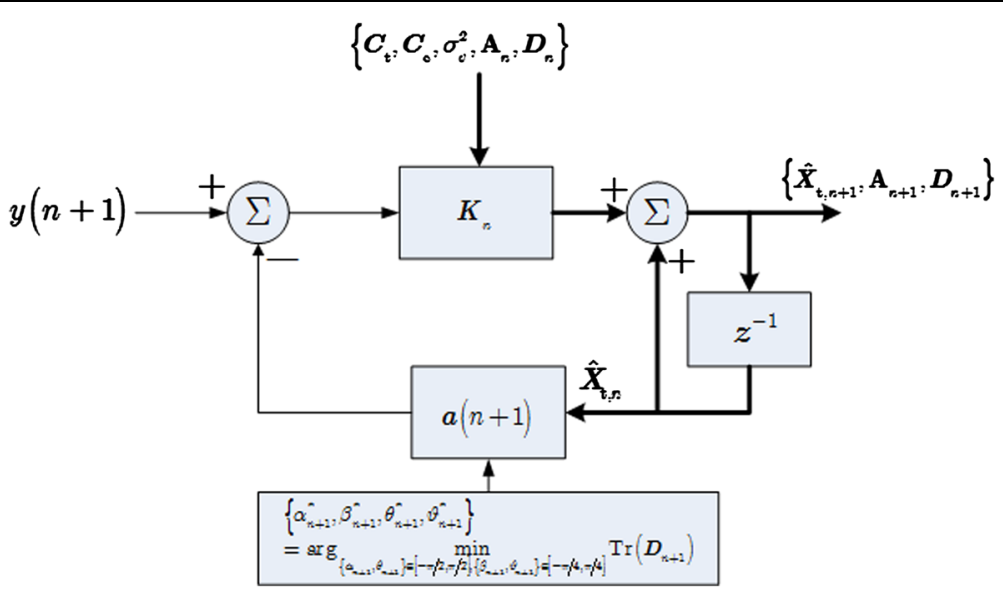

Fig. 1 Block diagram of the sequential estimation processing

complex envelope vector of noise for both polarizations, and the other parameters have the same definitions as in Eq. (1). This signal is sampled and passed through the matched filter whose output is appropriately normalized to move the effect of $\frac{g}{r^{2}}$ into the noise term. Finally, the normalized output of the matched filter at the receiver is given as

$$
\boldsymbol{y}=\left(\boldsymbol{S}_{\mathrm{t}}+\boldsymbol{S}_{\mathrm{c}}\right) \mathcal{\xi}+\boldsymbol{v}
$$

where $\boldsymbol{y}=\left[y_{\mathrm{h}} y_{\mathrm{v}}\right]^{\mathrm{T}}$ and $\boldsymbol{v}=\left[v_{\mathrm{h}} v_{\mathrm{v}}\right]^{\mathrm{T}}$. Stacking all the observations and the noise components into column vectors, in a similar fashion to the approach used in Section 2, we obtain $2 N$-dimensional vectors $\mathbf{y}^{\prime}$ and $\mathbf{v}^{\prime}$, i.e., $\quad \mathbf{y}^{\prime}=\left[y_{\mathrm{h}}(1), y_{\mathrm{v}}(1), \ldots, y_{\mathrm{h}}(N), y_{\mathrm{v}}(N)\right]^{\mathrm{T}} \quad$ and $\quad \mathbf{v}^{\prime}=$ $\left[v_{\mathrm{h}}(1), v_{\mathrm{v}}(1), \ldots, v_{\mathrm{h}}(N), v_{\mathrm{v}}(N)\right]^{\mathrm{T}}$, respectively. Vectors $\boldsymbol{X}_{\mathrm{t}}$ and $X_{\mathrm{c}}$ remain the same as defined earlier.

We also define the transformation matrix

$$
\mathbf{T}=\left[\begin{array}{cccc}
\xi_{\mathrm{h}}^{(1)} & \xi_{\mathrm{v}}^{(1)} & 0 & 0 \\
0 & 0 & \xi_{\mathrm{h}}^{(1)} & \xi_{\mathrm{v}}^{(1)} \\
& \vdots & & \\
\xi_{\mathrm{h}}^{(N)} & \xi_{\mathrm{v}}^{(N)} & 0 & 0 \\
0 & 0 & \xi_{\mathrm{h}}^{(N)} & \xi_{\mathrm{v}}^{(N)}
\end{array}\right]
$$

So the observation vector is expressed as

$$
\mathbf{y}^{\prime}=\mathbf{T} \boldsymbol{X}_{\mathrm{t}}+\mathbf{T} \boldsymbol{X}_{\mathrm{c}}+\mathbf{v}^{\prime}
$$

Thus, we obtain a similar linear model for the vector system with the scalar one. In order to make a fair comparison, assume that $v_{\mathrm{h}}(i), v_{\mathrm{v}}(i)$, and $i=1, \ldots, N$ have the same power with $\frac{\sigma^{2}}{2}$ and are uncorrelated with each other. Then, the covariance matrix $D^{\prime}$ of $X_{\mathrm{t}}^{\prime}$ 's minimum MSE estimation from $\mathbf{y}^{\prime}$ is

$$
\boldsymbol{D}^{\prime}=\left(\boldsymbol{C}_{\mathrm{t}}^{-1}+\mathbf{T}^{\mathrm{H}}\left(\mathbf{T} \boldsymbol{C}_{\mathrm{c}} \mathbf{T}^{\mathrm{H}}+\frac{\sigma_{v}^{2}}{2} \mathbf{I}_{2 N}\right)^{-1} \mathbf{T}\right)^{-1}
$$

Substituting the trigonometric representation of (14) into (24) and following the same way in Section 4.1, we can prove that the initial signal phase $\phi$ does not affect the value of $\boldsymbol{D}^{\prime}$. Since the relevant proof can be obtained similarly as that given for Property 1 , it is omitted for the sake of brevity. Hence, the optimization problem of seeking the optimal transmission polarization to minimize the MSE of estimating $\boldsymbol{X}_{\mathrm{t}}$ for vector systems can be formulated as

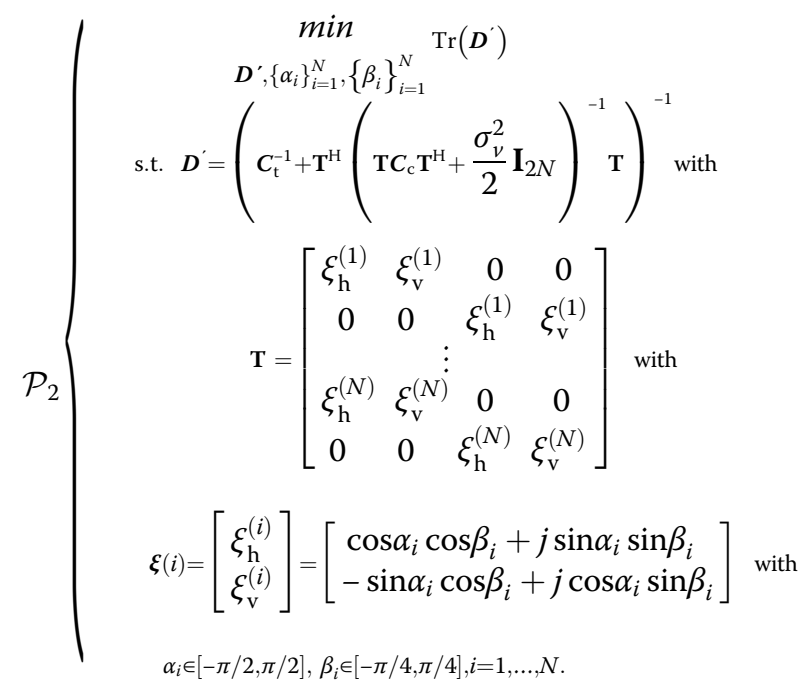

Similar in solving $\mathcal{P}_{1}$, the computational complexity of solving $\mathcal{P}_{2}$ depends exponentially on the observation number $N$, as well as the complexity burden required at each observation, i.e., the maximum point search algorithm used for parameters $\{\alpha, \beta\}$. Considering employing lattice search with $l_{\alpha}$ and $l_{\beta}$ points in each dimension of the domain space $\{\alpha, \beta\}$, the required complexity is $O\left(\left(l_{\alpha} l_{\beta}\right)^{N}\right)$. 


\subsection{Sequential estimation algorithm for vector systems}

Because of the similar reason, namely directly solving $\mathcal{P}_{2}$ by searching for the optimal trigonometric parameters with latter search requires a high complexity burden, now in this subsection, we develop the relevant sequential optimization procedure for such vector system. Let $\hat{\boldsymbol{X}}_{\mathrm{t}, n}$ denote the minimum MSE estimator based on the observations $\left[y_{\mathrm{h}}(1), y_{\mathrm{v}}(1), \ldots, y_{\mathrm{h}}(n), y_{\mathrm{v}}(n)\right]^{\mathrm{T}}$ and $\boldsymbol{D}_{n}^{\prime}$ be the corresponding covariance matrix of the estimator. Then, with the new sample $\boldsymbol{y}(n+1)$, the estimator is updated as

$$
\hat{\boldsymbol{X}}_{\mathrm{t}, n+1}=\hat{\boldsymbol{X}}_{\mathrm{t}, n}+\boldsymbol{K}_{n+1}\left(\boldsymbol{y}(n+1)-\boldsymbol{t}^{\mathrm{T}}(n+1) \hat{\boldsymbol{X}}_{\mathrm{t}, n}\right),
$$

where

$$
\boldsymbol{t}(n+1)=\left[\begin{array}{ccccc}
\xi_{\mathrm{h}}(n+1) & \xi_{\mathrm{v}}(n+1) & 0 & 0 \\
0 & 0 & \xi_{\mathrm{h}}(n+1) & \xi_{\mathrm{v}}(n+1)
\end{array}\right]^{\mathrm{T}}
$$

and

$$
\frac{\boldsymbol{K}_{n+1}=\boldsymbol{D}_{n}^{\prime} \boldsymbol{t}(n+1)}{{\left[\mathbf{T}_{n+1} \boldsymbol{C}_{\mathrm{c}} \mathbf{T}_{n+1}^{\mathrm{H}}+\frac{\sigma_{v}^{2}}{2} \mathbf{I}_{2(n+1)}\right]_{2(n+1), 2(n+1)}+\boldsymbol{t}^{\mathrm{H}}(n+1) \boldsymbol{D}_{n}^{\prime}}_{t}^{t}} \boldsymbol{t}(n+1),
$$

with $\mathbf{T}_{n+1}=[\boldsymbol{t}(1), \ldots, \boldsymbol{t}(n+1)]^{\mathrm{T}}$ and $[\cdot]_{2(n+1), 2(n+1)}$ denoting the $2(n+1)$ th diagonal element of matrix [.]. In the meantime, the covariance matrix is updated as

$$
\boldsymbol{D}_{n+1}^{\prime}=\left(\mathbf{I}_{2(n+1)}-\boldsymbol{K}_{n+1} \boldsymbol{t}^{\mathrm{T}}(n+1)\right) \boldsymbol{D}_{n}^{\prime}
$$

Again, as to $\boldsymbol{D}_{n+1}^{\prime}$, with $n$ increasing, $\left[\boldsymbol{D}_{n+1}^{\prime}\right]_{i i}$ decreases and converges to a certain value. As a consequence, $\operatorname{tr}\left(\boldsymbol{D}_{n+1}^{\prime}\right)$ is also a monotonic decreasing sequence and converges to a certain value. Finally, the devised sequential optimization procedure is summarized in Algorithm 2.
It is worth noticing that the computational complexity of Algorithm 2 is linear to the observation number $N$, with requiring the lattice search to seek the optimal polarization in the domain space $\{\alpha, \beta\}$ for every observation. Thus, the overall complexity is $O\left(l_{\alpha} l_{\beta} N\right)$. Compared to handling $\mathcal{P}_{2}$ straightly with lattice search (the corresponding computational complexity is $\left.O\left(\left(l_{\alpha} l_{\beta}\right)^{N}\right)\right)$, the computation burden is greatly reduced. The reason why the proposed algorithm is less expensive than solving $\mathcal{P}_{2}$ is similar as that in Algorithm 1, namely it is sequential and does not require optimization for all observations.

\section{Numerical examples}

In this section, the performance analysis of the proposed algorithms is presented. As to scalar measurement systems with the joint $\mathrm{Tx} / \mathrm{Rx}$ polarization optimization, we provide numerical examples to demonstrate the effectiveness of the devised algorithms, in comparison with two other polarization approaches. Additionally, we also compare their performances with that of the vector measurement system with transmission polarization optimization and highlight the advantage of the latter design.

Throughout the simulations, we generate the target and clutter covariance matrices employing a similar way as in [3]. Though the solution in [3] is criticized incorrectly in Section 1, the approach there to generate target and clutter covariance matrices is fine. Precisely, the target covariance matrix is chosen as

$$
C_{\mathrm{t}}=a \boldsymbol{U}_{\mathrm{t}} \Lambda_{\mathrm{t}} \boldsymbol{U}_{\mathrm{t}}^{\mathrm{H}}
$$

where $\boldsymbol{U}_{\mathrm{t}}$ is an arbitrary unitary matrix constructed with the left singular vectors of a $4 \times 4$ matrix $M$ with independent and identical distribution complex Gaussian entries, i.e., $\boldsymbol{M}=\boldsymbol{U}_{\mathrm{t}} \boldsymbol{\Lambda}_{M} \boldsymbol{U}_{\mathrm{r}}, \boldsymbol{\Lambda}_{\mathrm{t}}=\operatorname{diag}(\operatorname{rand}(1,4))$ with $\operatorname{rand}(1$, 4) denoting a random four-dimensional real vector, and $\operatorname{diag}(\cdot)$ being the diagonalization of the vector (.). Note that we normalize rand $(1,4)$ to keep the target power to be constant. $a$ is the factor that controls the

Algorithm 2: compute the optimal transmission polarization for vector measurement systems

$$
\begin{aligned}
& \text { Input: } \boldsymbol{C}_{\mathrm{t}}, \boldsymbol{C}_{\mathrm{c}}, \sigma_{v}^{2}, \boldsymbol{y}_{n+1}, \mathbf{T}_{n}, \boldsymbol{D}_{n}^{\prime} \\
& \text { 1: find }\left\{\alpha_{n+1}^{*}, \beta_{n+1}^{*}\right\}=\arg \min _{\left\{\alpha_{n+1}, \beta_{n+1}\right\} \in[-\pi / 2, \pi / 2] \times[-\pi / 4, \pi / 4]} \operatorname{Tr}\left(\boldsymbol{D}_{n+1}^{\prime}\right) \text {, where } \boldsymbol{D}_{n+1}^{\prime} \text { is given in Eq. } \\
& \text { 2: Set } \boldsymbol{t}(n+1) \text { based on } \alpha_{n+1}^{*}, \beta_{n+1}^{*} . \\
& \text { 3: Update } \hat{\boldsymbol{X}}_{\mathrm{t}} \text { using Eq. (26). }
\end{aligned}
$$


signal-to-clutter-plus-noise ratio (SCNR) with the definition [5]:

$$
\mathrm{SCNR}=\frac{\operatorname{tr}\left(\boldsymbol{C}_{\mathrm{t}}\right)}{\operatorname{tr}\left(\boldsymbol{C}_{\mathrm{c}}\right)+\sigma_{v}^{2}},
$$

where the clutter covariance matrix is chosen as

$$
\boldsymbol{C}_{\mathrm{c}}=\boldsymbol{U}_{\mathrm{c}} \boldsymbol{\Lambda}_{\mathrm{c}} \boldsymbol{U}_{\mathrm{c}}^{\mathrm{H}}
$$

with $\boldsymbol{\Lambda}_{\mathrm{c}}=\operatorname{diag}([0.25,0.25,0.25,0.25])$ and $\boldsymbol{U}_{\mathrm{c}}$ being chosen with the similar operation to generate $\boldsymbol{U}_{\mathrm{t}}$. Throughout the simulations, we assume the noise power $\sigma_{v}^{2}=0 \mathrm{~dB}$.

Furthermore, by inspection of Eqs. (19) and (29), we can observe that $\boldsymbol{D}_{n+1}$ and $\boldsymbol{D}_{n+1}^{\prime}$ do not depend on the observations $\left\{y_{i+1}\right\}_{i=0}^{n}$ and $\left\{\boldsymbol{y}_{i+1}\right\}_{i=0}^{n}$, respectively, even though in Algorithm 1 and Algorithm 2, both of them are the input parameters that update the estimation of target scattering vector $\hat{\boldsymbol{X}}_{\mathrm{t}}$. Since the MSE of $\hat{\boldsymbol{X}}_{\mathrm{t}}$ is the figure of merit for the optimization problem and $\operatorname{Tr}\left(\boldsymbol{D}_{n+1}\right), \operatorname{Tr}$ $\left(\boldsymbol{D}_{n+1}^{\prime}\right)$ are the objectives to be optimized for each algorithm, respectively, in this section, we will investigate the changes of $\operatorname{Tr}\left(\boldsymbol{D}_{n+1}\right)$ and $\operatorname{Tr}\left(\boldsymbol{D}_{n+1}^{\prime}\right)$ without considering updating of $\hat{X}_{\mathrm{t}}$. As a consequence, we do not use $\left\{y_{i+1}\right\}_{i=0}^{n}$ and $\left\{\boldsymbol{y}_{i+1}\right\}_{i=0}^{n}$ in the simulation. Also notice that $n \geq 4$ is required for both the proposed algorithms. So we randomly choose $\left\{\alpha_{i}\right\}_{i=1}^{4}=\left\{\beta_{i}\right\}_{i=1}^{4}=\left\{\theta_{i}\right\}_{i=1}^{4}=\left\{\vartheta_{i}\right\}_{i=1}^{4}=\pi / 8$ (some other values within the definition ranges can be also used) as the initial trigonometric parameters to generate $\mathbf{A}_{4}$ and $\mathbf{T}_{4}$ for Algorithm 1 and Algorithm 2, respectively.

Finally, regarding the lattice search used to seek the optimal polarizations for Algorithm 1 and Algorithm 2, set $l_{\alpha}=l_{\theta}=10^{3}$ and $l_{\beta}=l_{9}=500$. By using the Monte Carlo method, the MSEs are calculated by averaging $10^{5}$ independent realizations of $\boldsymbol{C}_{\mathrm{t}}$ and $\boldsymbol{C}_{\mathrm{c}}$, respectively.

\subsection{Scalar measurement systems}

We choose the conventional polarimetric radar and the polarimetric radar with adaptive transmission polarization as the counterparts to compare their performances with the polarimetric radar with joint $\mathrm{Tx} / \mathrm{Rx}$ optimization. As to conventional polarimetric systems, the transmission side alternatively transmits horizontal and vertical polarizations, i.e., $\boldsymbol{\xi}=[1,0]^{\mathrm{T}}$ in the current pulse and $\boldsymbol{\xi}=[0,1]^{\mathrm{T}}$ in next pulse. In the reception side, the horizontal and vertical polarizations are simultaneously received, so the reception vector satisfies $\boldsymbol{\eta}=[\sqrt{2} / 2, \sqrt{2} / 2]^{\mathrm{T}}$. Therefore, the relevant MSE can be calculated by substituting the waveform parameters $\{\alpha=0, \beta=0, \theta=-\pi / 4, \vartheta=0\}$ and $\{\alpha=-\pi / 2, \beta=$ $0, \theta=-\pi / 4,9=0\}$, alternatively, into Algorithm 1 with the increase of observation samples and without optimal polarization search. Then, as to the scalar system with transmission polarization optimization, the transmission polarization $\left[\xi_{\mathrm{h}}, \xi_{\mathrm{v}}\right]^{\mathrm{T}}$ is allowed to be chosen freely while the reception polarization is fixed as $[\sqrt{2} / 2, \sqrt{2} / 2]^{\mathrm{T}}$. As such, we can employ Algorithm 1 to optimally select transmission polarization by fixing $\theta=-\pi / 4$ and $\vartheta=0$.

By employing Algorithm 1, Fig. 2 plots the MSEs of the estimating target scattering matrix $S_{\mathrm{t}}$ versus the number of observation samples for the aforementioned three scalar systems, with $\mathrm{SCNR}=0 \mathrm{~dB}$. As expected, the devised method monotonically reduces the MSE and converges to a certain value. On the other hand, the plots clearly show that the scalar system with joint $T x \backslash R x$ polarization optimization performs better than that with transmission polarization optimization as well as the scalar system with transmission polarization optimization showing a significant performance gain with respect to conventional polarization radars.

Additionally, Fig. 3 draws the MSE of the estimating target scattering matrix $S_{\mathrm{t}}$ versus the SCNR, with a fixed 50 observation samples. It can be observed that, the scalar system with optimally designed $\mathrm{Tx} / \mathrm{Rx}$ polarizations leads to a power gain of $4-6 \mathrm{~dB}$ with respect to that with only transmission polarization optimization, as well as $6-8 \mathrm{~dB}$ gain with respect to the conventional polarization radar.

\subsection{Scalar measurement systems versus vector measurement systems}

In this subsection, we compare the performance of scalar measurement systems with that of vector systems. As in Fig. 4, the MSEs of the three aforementioned scalar systems and the vector system with transmission polarization optimization versus the number of observation samples with $\mathrm{SCNR}=0 \mathrm{~dB}$ are illustrated. The results highlight that the vector measurement with transmission polarization optimization has almost the same estimation performance as the scalar measurement with joint $\mathrm{Tx} / \mathrm{Rx}$ polarization

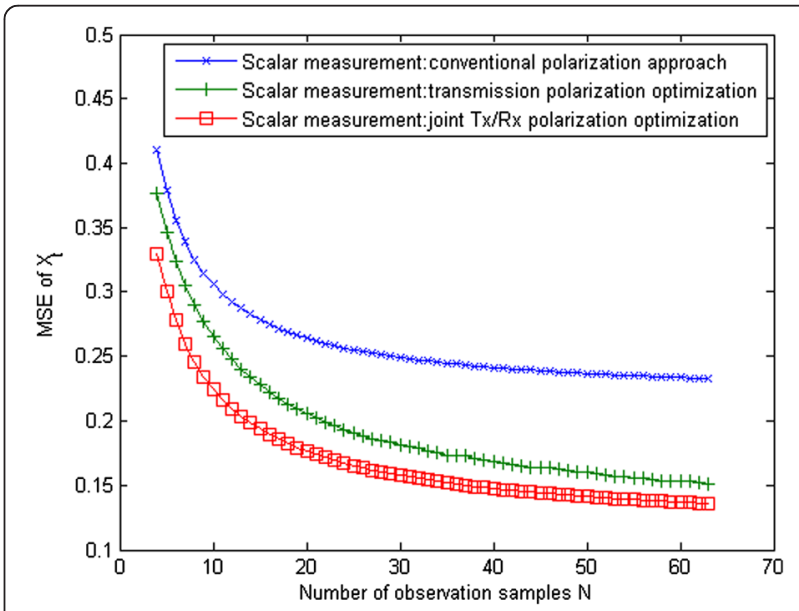

Fig. 2 MSE performance comparison of estimating the target scattering matrix $\boldsymbol{S}_{\mathrm{t}}$ versus the number of observation samples, with $\mathrm{SCNR}=0 \mathrm{~dB}$ 


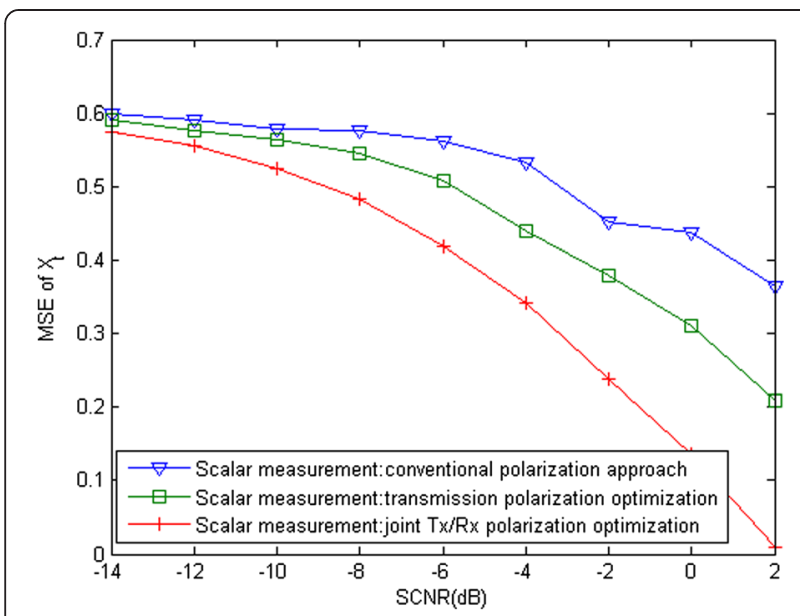

Fig. 3 MSE performance comparison of estimating the target scattering matrix $\boldsymbol{S}_{\mathrm{t}}$ versus the SCNR, with a fixed 50 observation samples

optimization. This is because, in the former case, the receiver polarization optimization is implicitly performed. In other words, even though we perform joint optimization over both transmission and reception polarizations for the scalar system, we are still finding the best linear combination of the two received measurements at each receiver. However, combining them linearly need not be the overall optimal solution, even if we spend much more time on doing so. This can be avoided by retaining the vector measurements, thereby showing better performance as demonstrated in [6].

Finally, Fig. 5 shows the performance of the four systems as a function of SCNR, with the fixed length of observation sample. Again, the results demonstrate that the vector measurement system has the same performance with the scalar measurement system with joint $\mathrm{Tx} / \mathrm{Rx}$ optimization.

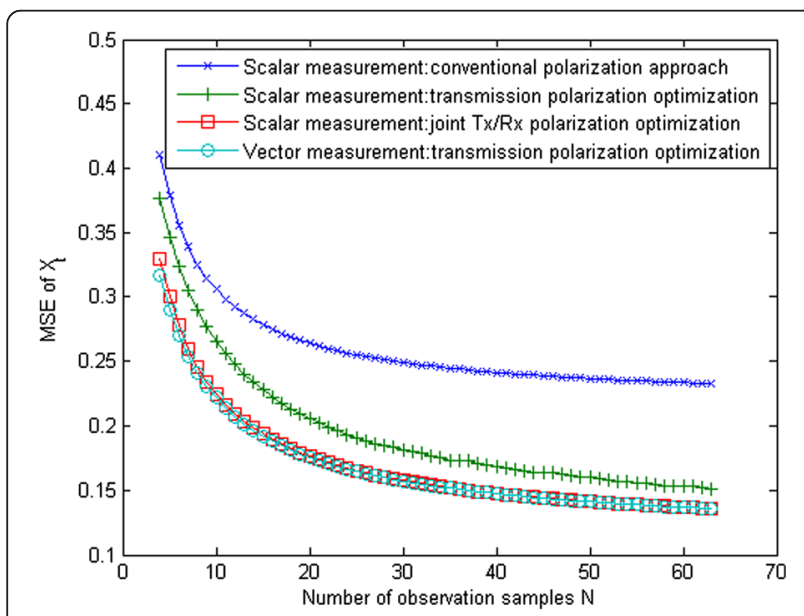

Fig. 4 MSE performance comparison between scalar measurement systems and vector measurement ones versus the number of observation samples, with SCNR $=0 \mathrm{~dB}$

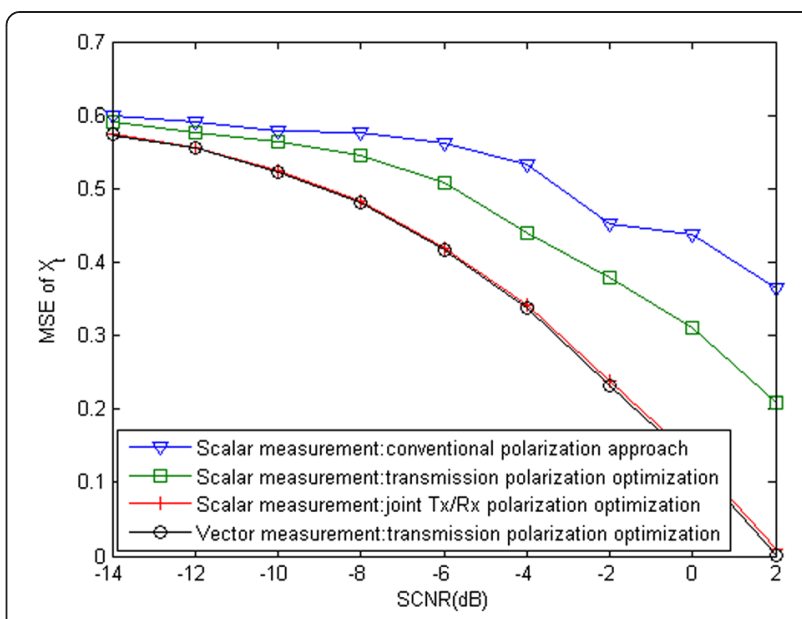

Fig. 5 MSE performance comparison between scalar measurement systems and vector measurement ones versus the SCNR, with a fixed 50 observation samples

\section{Conclusions}

Adaptive polarization design of polarimetric radars, for the purpose of optimally estimating the target polarization scattering vector, is studied. Starting from the problem of designing the optimal polarizations for the scalar measurement system with joint $\mathrm{Tx} / \mathrm{Rx}$ polarization optimization, under considering the minimum MSE of the estimator as the figure of merit, a sequential method of selecting the optimal Tx/Rx polarizations for such system is developed by owing to a suitable reformulation of the considered non-convex design problem. Furthermore, by employing a similar deviation procedure, a sequential method to select the optimal transmission polarization for the vector system with transmission polarization optimization is also devised. Since both the proposed algorithms make use of the sequential minimum MSE estimation, they monotonically decrease the MSE of the estimation and converge to a stationary point. The complexity of the proposed methods is linear with the number of outer iterations whereas at each iteration, it mainly requires the lattice search along four and two trigonometric parameters, respectively.

Several numerical examples have been provided to assess the effectiveness of the proposed methods. Precisely, the performance of the scalar measurement system with joint $\mathrm{Tx} \backslash \mathrm{Rx}$ polarization optimization, the one with transmission polarization optimization and the one with conventional polarimetric design, is compared. The system with joint $\mathrm{Tx} \backslash \mathrm{Rx}$ polarization optimization shows a significant performance improvement with respect to the one with transmission polarization optimization, as well as the conventional polarization approach. Moreover, the numerical 
results also show that the vector measurement system with transmission polarization optimization provides the comparative estimation performance with the scalar measurement system with joint $\mathrm{Tx} / \mathrm{Rx}$ optimization. This is because, in the latter case, the receiver polarization optimization is implicitly performed. But the vector measurement system requires a lower computation burden, highlighting the advantage of such system design.

\section{Appendix 1: Proof of the failure of the proposed method in [3] to solve Tx/Rx polarizations}

Before the proof, in such context, we declare that the parameter definitions and notations hereinafter have the same meaning as in [3]. In [3], the authors propose an adaptive waveform polarization method for the estimation of target scatter in the presence of clutter. The proposed algorithm, to determine the coefficients of the antenna transmission and reception polarizations, has a convex form and can be solved by employing SDP. However, the authors do not investigate whether the polarization vectors can be obtained after the convex problem is solved to obtain A (at least such processing is absent in the paper). In this appendix, we provide a proof that the transmission and reception polarization vectors cannot be effectively obtained through the solution of A. Essentially, the convex form is only a relaxation version of the initial problem. The proof is as follows.

Let us assume that $\mathbf{A}$ is already solved by employing the proposed convex optimization in [3]. A is an $m \times 4$ complex matrix and based on (4) and (6) of [3], its $i$ th row satisfies

$$
\begin{aligned}
\mathbf{a}_{i} i & =\mathbf{a}(P(i) ; \boldsymbol{\xi}(i), \boldsymbol{\eta}(i)) \\
= & \sqrt{P(i)} \mathbf{p}(\boldsymbol{\xi}(i), \boldsymbol{\eta}(i)) \\
\triangleq \sqrt{P(i)} & {\left[\left(\xi_{\mathrm{h}}^{(i)}\right)^{*} \eta_{\mathrm{h}}^{(i)}\left(\xi_{\mathrm{v}}^{(i)}\right)^{*} \eta_{\mathrm{v}}^{(i)}\left(\xi_{\mathrm{h}}^{(i)}\right)^{*} \eta_{\mathrm{v}}^{(i)}\left(\xi_{\mathrm{v}}^{(i)}\right)^{*} \eta_{\mathrm{h}}^{(i)}\right]^{\mathrm{T}} . }
\end{aligned}
$$

Notice that the transmission polarization vector $\boldsymbol{\xi}$ can be generally parameterized as follow:

$$
\begin{aligned}
& \boldsymbol{\xi}=\left[\begin{array}{ll}
\xi_{\mathrm{h}} & \xi_{\mathrm{v}}
\end{array}\right]^{\mathrm{H}} \triangleq\left[\begin{array}{ll}
\cos \phi \mathrm{e}^{j \theta_{\mathrm{h}}} & \sin \phi \mathrm{e}^{j \theta_{\mathrm{v}}}
\end{array}\right] \\
& \left\{\begin{array}{l}
\phi=0.25 \times\left(\arccos \left(p_{i 1}+p_{i 2}\right)+\arccos \left(p_{i 1}-p_{i 2}\right)+\arcsin \left(p_{i 4}-p_{i 3}\right)+\arcsin \left(p_{i 3}+p_{i 4}\right)\right) \\
\phi=0.25 \times\left(\arccos \left(p_{i 1}-p_{i 2}\right)-\arccos \left(p_{i 1}+p_{i 2}\right)+\arcsin \left(p_{i 3}+p_{i 4}\right)-\arcsin \left(p_{i 4}-p_{i 3}\right)\right)
\end{array} .\right.
\end{aligned}
$$

where $\left\{\phi, \theta_{\mathrm{h}}, \theta_{\mathrm{v}}\right\} \in(-\pi, \pi]$. In a similar way, the reception polarization vector $\boldsymbol{\eta}$ can be taken into the following form:

$$
\boldsymbol{\eta}=\left[\eta_{\mathrm{h}} \eta_{\mathrm{v}}\right]^{\mathrm{H}} \triangleq\left[\begin{array}{ll}
\cos \phi \mathrm{e}^{j \vartheta_{\mathrm{h}}} & \sin \phi \mathrm{e}^{\mathrm{j} \vartheta_{\mathrm{v}}}
\end{array}\right],
$$

where $\left\{\phi, \vartheta_{\mathrm{h}}, \vartheta_{\mathrm{v}}\right\} \in(-\pi, \pi]$. Moreover, we also introduce parameterized representation of the $4 \times 1$ complex vector $\mathbf{p}(\boldsymbol{\xi}(i), \boldsymbol{\eta}(i))$ as follow:

$$
\mathbf{p}(\boldsymbol{\xi}(i), \boldsymbol{\eta}(i)) \triangleq\left[p_{i 1} \mathrm{e}^{j \beta_{i 1}}, p_{i 2} \mathrm{e}^{j \beta_{i 2}}, p_{i 3} \mathrm{e}^{j \beta_{i 3}}, p_{i 4} \mathrm{e}^{j \beta_{i 4}}\right] .
$$

where $p_{i 1}^{2}+p_{i 2}^{2}+p_{i 3}^{2}+p_{i 4}^{2}=1$ and $\left\{\beta_{i, 1}, \beta_{i, 2}, \beta_{i, 3}, \beta_{i, 4}\right\} \in$ $(-\pi, \pi]$.

Hence, by introducing (34), (35), and (36) into (33), we obtain the following equations:

$$
\left\{\begin{array}{l}
\left(\xi_{\mathrm{h}}^{(i)}\right)^{*} \eta_{\mathrm{h}}^{(i)}=\cos \phi \cos \phi \mathrm{e}^{j\left(\vartheta_{\mathrm{h}}-\theta_{\mathrm{h}}\right)}=p_{i 1} \mathrm{e}^{j \beta_{i 1}} \\
\left(\xi_{\mathrm{v}}^{(i)}\right)^{*} \eta_{\mathrm{v}}^{(i)}=\sin \phi \sin \phi \mathrm{e}^{j\left(\vartheta_{\mathrm{v}}-\theta_{\mathrm{v}}\right)}=p_{i 2} \mathrm{e}^{\mathrm{j} \beta_{i 2}} \\
\left(\xi_{\mathrm{h}}^{(i)}\right)^{*} \eta_{\mathrm{v}}^{(i)}=\cos \phi \sin \phi \mathrm{e}^{j\left(\vartheta_{\mathrm{v}}-\theta_{\mathrm{h}}\right)}=p_{i 3} \mathrm{e}^{j \beta_{i 3}} \\
\left(\xi_{\mathrm{v}}^{(i)}\right)^{*} \eta_{\mathrm{h}}^{(i)}=\sin \phi \cos \phi \mathrm{e}^{j\left(\vartheta_{\mathrm{h}}-\theta_{\mathrm{v}}\right)}=p_{i 4} \mathrm{e}^{j \beta_{i 4}}
\end{array}\right.
$$

Furthermore, (37) can be recast into two equation systems, namely

$$
\left\{\begin{array}{l}
\cos \phi \cos \phi=p_{i 1} \\
\sin \phi \sin \phi=p_{i 2} \\
\cos \phi \sin \phi=p_{i 3} \\
\sin \phi \cos \phi=p_{i 4}
\end{array},\right.
$$

and

$$
\left\{\begin{array}{l}
\vartheta_{\mathrm{h}}-\theta_{\mathrm{h}}=\beta_{i 1} \\
\vartheta_{\mathrm{v}}-\theta_{\mathrm{v}}=\beta_{i 2} \\
\vartheta_{\mathrm{v}}-\theta_{\mathrm{h}}=\beta_{i 3} \\
\vartheta_{\mathrm{h}}-\theta_{\mathrm{v}}=\beta_{i 4}
\end{array}\right.
$$

As to (38), after some algebraic manipulations and inverse trigonometrical transform, we can transform (38) into the following equations:

$$
\left\{\begin{array}{l}
\phi-\phi=\arccos \left(p_{i 1}+p_{i 2}\right) \\
\phi+\phi=\arccos \left(p_{i 1}-p_{i 2}\right) \\
\phi+\phi=\arcsin \left(p_{i 3}+p_{i 4}\right) \\
\phi-\phi=\arcsin \left(p_{i 4}-p_{i 3}\right)
\end{array} .\right.
$$

We can see that (40) is an over-determined equation system. By employing the least-square method, we can search for the "approximately" solution of $\phi$ 
As to (39), after some algebraic manipulations, we obtain the following equation system:

$$
\left\{\begin{array}{l}
\theta_{\mathrm{h}}-\theta_{\mathrm{v}}=\beta_{i 4}-\beta_{i 1} \\
\theta_{\mathrm{h}}-\theta_{\mathrm{v}}=\beta_{i 2}-\beta_{i 3} \\
\vartheta_{\mathrm{h}}-\vartheta_{\mathrm{v}}=\beta_{i 1}-\beta_{i 3} \\
\vartheta_{\mathrm{h}}-\vartheta_{\mathrm{v}}=\beta_{i 4}-\beta_{i 2}
\end{array}\right.
$$

Since $\beta_{i 1}, \beta_{i 2}, \beta_{i 3}$, and $\beta_{i 4}$ can be, respectively, an arbitrary value within $(-\pi, \pi]$, the first equation may conflict with the second equation when $\beta_{i 4}-\beta_{i 1} \neq \beta_{i 2}$ $-\beta_{i 3}$ and the third one may contradict the fourth one when $\beta_{i 1}-\beta_{i 3} \neq \beta_{i 4}-\beta_{i 2}$.

As a consequence, the transmission and reception polarization vectors cannot be always effectively solved based on $\mathbf{A}$. The proof is concluded.

RemarksLemma 2.1 of [10] provides a three-parameter representation of the signal polarization, which has a different form from our so-calledtwo-parameter "general" formulation (34). In the appendix, we do not implement such three-parameter representation since the proof will become more cumbersome and prolix by employing the new one. But the study shows (not shown here) that the question of conflict arises again with the use of the threeparameter representation.

\section{Appendix 2: Proof of Property 1}

Let us first introduce the following new variables for (11)

$$
\left\{\begin{array}{l}
\boldsymbol{B}=\sigma_{v}^{-1} \mathbf{A} C_{\mathrm{c}}^{1 / 2} \\
\boldsymbol{C}_{\mathrm{t}, 1}^{-1}=\boldsymbol{C}_{\mathrm{c}}^{1 / 2} \boldsymbol{C}_{\mathrm{t}}^{-1} \boldsymbol{C}_{\mathrm{c}}^{1 / 2} \\
\boldsymbol{D}_{1}^{-1}=\boldsymbol{C}_{\mathrm{c}}^{1 / 2} \boldsymbol{D}^{-1} \boldsymbol{C}_{\mathrm{c}}^{1 / 2}
\end{array}\right.
$$

Then (11) can be written into

$$
\boldsymbol{D}_{1}^{-1}=\boldsymbol{C}_{\mathrm{t}, 1}^{-1}+\boldsymbol{B}^{\mathrm{H}}\left(\boldsymbol{B} \boldsymbol{B}^{\mathrm{H}}+\mathbf{I}_{N}\right)^{-1} \boldsymbol{B} .
$$

Using the matrix inversion lemma, we obtain

$$
\left(\mathbf{I}_{4}+\boldsymbol{B}^{\mathrm{H}} \boldsymbol{B}\right)^{-1}=\mathbf{I}_{4}-\boldsymbol{B}^{\mathrm{H}}\left(\mathbf{I}_{N}+\boldsymbol{B} \boldsymbol{B}^{\mathrm{H}}\right)^{-1} \boldsymbol{B} .
$$

Therefore, we have

$$
\boldsymbol{D}_{1}^{-1}=\boldsymbol{C}_{\mathrm{t}, 1}^{-1}+\mathbf{I}_{4}-\left(\mathbf{I}_{4}+\boldsymbol{B}^{\mathrm{H}} \boldsymbol{B}\right)^{-1},
$$

Meanwhile, substituting (14) and (15) into the second term of (8), the following equation is obtained

$$
\mathbf{A}=\mathbf{U} \boldsymbol{M},
$$

where

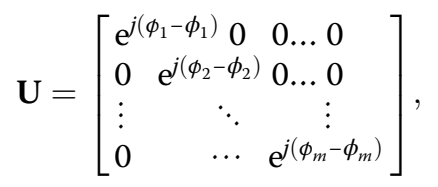

and

$$
\boldsymbol{M}=\left[\begin{array}{l}
\zeta_{\mathrm{h}}^{(1)} \iota_{\mathrm{h}}^{(1)}, \zeta_{\mathrm{h}}^{(1)} \iota_{\mathrm{v}}^{(1)}, \zeta_{\mathrm{v}}^{(1)} \iota_{\mathrm{h}}^{(1)}, \zeta_{\mathrm{v}}^{(1)} \iota_{\mathrm{v}}^{(1)} \\
\vdots \\
\zeta_{\mathrm{h}}^{(N)} \iota_{\mathrm{h}}^{(N)}, \zeta_{\mathrm{h}}^{(N)} \iota_{\mathrm{v}}^{(N)}, \zeta_{\mathrm{v}}^{(N)} \iota_{\mathrm{h}}^{(N)}, \zeta_{\mathrm{v}}^{(N)} \iota_{\mathrm{v}}^{(N)}
\end{array}\right] .
$$

$\mathbf{U}$ is a unitary matrix, and $\mathbf{U}^{\mathrm{H}} \mathbf{U}=\mathbf{U U}^{\mathrm{H}}=\mathbf{I}_{N}$. Thus, the following equation holds

$$
\begin{aligned}
& \boldsymbol{B}^{\mathrm{H}} \boldsymbol{B}=\sigma_{v}^{-1}\left(\boldsymbol{C}_{\mathrm{c}}^{1 / 2}\right)^{\mathrm{H}} \mathbf{A}^{\mathrm{H}} \cdot \sigma_{v}^{-1} \mathbf{A} \boldsymbol{C}_{\mathrm{c}}^{1 / 2} \\
& =\sigma_{v}^{-2}\left(\boldsymbol{C}_{\mathrm{c}}^{1 / 2}\right)^{\mathrm{H}} \boldsymbol{M}^{\mathrm{H}} \mathbf{U}^{\mathrm{H}} \mathbf{U} \boldsymbol{M} \boldsymbol{C}_{\mathrm{c}}^{1 / 2} \\
& =\sigma_{v}^{-2}\left(\boldsymbol{C}_{\mathrm{c}}^{1 / 2}\right)^{\mathrm{H}} \boldsymbol{M}^{\mathrm{H}} \boldsymbol{M} \boldsymbol{C}_{\mathrm{c}}^{1 / 2}
\end{aligned}
$$

Noticing that

$$
\operatorname{Tr}(\boldsymbol{D})=\operatorname{Tr}\left(\boldsymbol{C}_{\mathrm{c}}^{1 / 2} \boldsymbol{D}_{1} \boldsymbol{C}_{\mathrm{c}}^{1 / 2}\right)=\operatorname{Tr}\left(\boldsymbol{C}_{\mathrm{c}} \boldsymbol{D}_{1}\right),
$$

substituting (44) and (50) into (51), the trace of $\boldsymbol{D}$ can be written as

$$
\begin{aligned}
\operatorname{Tr}(\boldsymbol{D}) & =\operatorname{Tr}\left(\boldsymbol{C}_{\mathrm{c}} \boldsymbol{D}_{1}\right) \\
& =\operatorname{Tr}\left(\boldsymbol{C}_{\mathrm{c}}\left(\boldsymbol{C}_{\mathrm{t}, 1}^{-1}+\mathbf{I}_{4}-\left(\mathbf{I}_{4}+\sigma_{v}^{-2}\left(\boldsymbol{C}_{\mathrm{c}}^{1 / 2}\right)^{\mathrm{H}} \boldsymbol{M}^{\mathrm{H}} \boldsymbol{M} \boldsymbol{C}_{\mathrm{c}}^{1 / 2}\right)^{-1}\right)^{-1}\right) .
\end{aligned}
$$

Therefore, the initial signal phases $\phi$ and $\phi$ do not affect the value of $\boldsymbol{D}$. This concludes the proof.

\section{Competing interests}

The authors declare that they have no competing interests.

\section{Acknowledgements}

The authors would like to thank Mr. John Lucynski for providing some comments on how to correct the language. This work was also supported by the National Natural Science Foundation of China (Nos. 61401488, 61501475, and 61490692).

Received: 28 March 2015 Accepted: 12 November 2015 Published online: 18 December 2015

\section{References}

1. D Giuli, Polarization diversity in radars. Proc. IEEE 74(2), 245-269 (1986)

2. J Wang, A Nehorai, Adaptive polarimetry design for a target in compound-Gaussian clutter. Signal Process. 89, 1061-1069 (2009)

3. J-J Xiao, A Nehorai, Joint transmitter and receiver polarization optimization for scattering estimation in clutter. IEEE Trans. Signal Process. 57(10), 4142-4147 (2009)

4. DA Garren, AC Odom, SU Pillai, JR Guerci, Full-polarization matched-illumination for target detection and identification. IEEE Trans. Aerosp. Electr. Syst. 38(3), 824-837 (2002)

5. M Hurtado, A Nehorai, Polarimetric detection of targets in heavy inhomogeneous clutter. IEEE Trans. Signal Process. 56(4), 1349-1361 (2008)

6. S Gogineni, A Nehorai, Polarimetric MIMO radar with distributed antennas for target detection. IEEE Trans. Signal Process. 58(3), 1689-1697 (2010)

7. J Liu, Z Zhang, Y Yang, Performance enhancement of subspace detection with a diversely polarized antenna. IEEE Signal Process. Lett. 19(1), 4-7 (2012)

8. J Liu, Z Zhang, Y Yang, Optimal waveform design for generalized likelihood ratio and adaptive matched filter detectors using a diversely polarized antenna. Signal Process. 92(4), 1126-1131 (2012)

9. M Hurtado, T Zhao, A Nehorai, Adaptive polarized waveform design for target tracking based on sequential Bayesian inference. IEEE Trans. Signal Process. 56(3), 1120-1133 (2008)

10. A Nehorai, E Paldi, Vector-sensor array processing for electromagnetic source localization. IEEE Trans. Signal Process. 42(2), 376-398 (1994)

11. S Boyd, L Vandenberghe, Semidefinite programming. SIAM Rev. 38, 49-95 (1996) 
12. CVX Research, Inc. CVX: Matlab software for disciplined convex programming, version 2.0. http://cvxr.com/cvx, April 2011

13. M A Richards, J A Scheer, W A Holm, Principles of Modern Radar: Basic principles (IET Digital Library, 2010)

14. A Aubry, A De Maio, A Farina, M Wicks, Knowledge-aided (potentially cognitive) transmit signal and receive filter design in signal-dependent clutter. IEEE Trans. Aerosp. Electron. Syst. 49(1), 93-117 (2013)

15. F Gini, A De Maio, L Patton, Waveform design and diversity for advanced radar systems (Institution of Engineering and Technology, London, 2012)

16. A Aubry, A De Maio, M Piezzo, A Farina, M Wicks, Cognitive design of the receive filter and transmitted phase code in reverberating environment. IET Radar Sonar Navig. 6(9), 822-833 (2012)

17. SM Kay, Fundamentals of statistical signal processing: estimation theory (Prentice-Hall, New Jersey, 1993)

18. A Nehorai, E Paldi, Acoustic vector-sensor array processing. IEEE Trans. Signal Process. 42(9), 2481-2491 (1994)

19. GE Box, WG Hunter, JS Hunter, Statistics for experimenters (John Wiley \& Sons, New York, 1978)

Submit your manuscript to a SpringerOpen ${ }^{\circ}$ journal and benefit from:

- Convenient online submission

- Rigorous peer review

- Immediate publication on acceptance

- Open access: articles freely available online

- High visibility within the field

- Retaining the copyright to your article

Submit your next manuscript at $>$ springeropen.com 